\title{
NEONATOLOGY: EVOLUTION FROM THE PAST TO FUTURE PERSPECTIVES
}

\section{Elisa Laschi, Serafina Perrone, Chiara Lembo, Giuseppe Buonocore}

\section{Department of Molecular and Developmental Medicine, University of Siena, Italy}

\begin{abstract}
The begin of modern neonatology takes place in the 1940s, when physicians first started to have interest in the newborn so that the primary responsibility for the neonate passed from the obstetricians to the neonatologists. In the $19^{\text {th }}$ century the term premature grouped together the concept of "preterm and weak infants", meant as babies suffering from poor energy and vitality. The idea that premature infants could be treated was introduced in the second half of the 19th century, when crucial fields signed the basis for neonatal care over the last century, such as thermoregulation, Apgar score, respiratory support, prenatal corticosteroids, metabolic screening and jaundice. From then on, advances in neonatology have resulted in the reduction of infant mortality worldwide. To date, scientific evidences have shown that the environmental conditions experienced in early life can profoundly influence human biology and long-term health. Chemical contaminants in water and diet, tobacco smoke, air pollution, gestational diabetes, hypertension and pre-eclampsia are all conditions that lead to the lowest common denominator oxidative stress. Fetuses and newborns -especially preterm- are particularly susceptible to oxidative stress mediated damage. Recently, the "omics" sciences represent the major area of growing interest and research in neonatology. The analysis of the metabolic profile detectable in a human biological fluid allows to instantly identifying changes in the composition of endogenous and exogenous metabolites caused by the interaction between specific physiopathological states, gene expression, and environment. From metabolomics studies comes the need of individualized and tailored medicine.
\end{abstract}

Key words: Newborn infant, Preterm newborn, Perinatal care, Metabolomics, Oxidative stress.

\section{SCIENTIFIC ACHIEVEMENTS FROM THE PAST}

In Biblical times, women were assisted by other women to stand on two bricks that were placed underneath their feet; the stones were dubbed "birthing bricks" and helped the midwife or assistant to have a little extra room to catch the baby. In the middle age, exclusively midwives assisted the births because men generally considered it uncouth to set foot in the delivery room. Of course this included the doctors, since they were men. ${ }^{1}$ The best-selling midwifery handbook of the time "Rose Garden for Pregnant Women and Midwives", came out in 1513, offering advice on such things as removing a stillborn. Sitting on a birthing stool - basically a horseshoe-shaped wood chair without a seat - was advised for delivery. ${ }^{2}$ In the 17 th Century, 3 to 5 women shared a bed at the famous Paris hospital Hotel Dieu: "if a woman died in childbirth, the other ones waited hours or even overnight for the orderly to cart the corpse away," Epstein writes in Get Me Out. ${ }^{3}$ The begin of modern neonatology takes place in the 1940s, when physicians first started to have interest in the newborn so that the primary responsibility for the neonate passed from the obstetricians to the neonatologists. ${ }^{4}$ In the $19^{\text {th }}$ century the term "premature" grouped together the concept of "preterm and weak infants", meant as babies suffering from poor energy and vitality. Many physicians had the idea that premature birth was the way of nature of expelling a defective fetus. ${ }^{5}$ Almost always newborns were born at home, lying-in hospitals were only used if the mother was destitute. Over the course of the 19th century obstetricians focus their attention on the mother and rarely on the infant beyond initial resuscitation. ${ }^{6}$ The idea that premature infants could be treated was probably introduced in the second half of the 19th century. The term neonatology was used for the first time in 1960 in the introduction of the first edition of the book Diseases of the Newborn by Alexander Schaffer. ${ }^{7}$ From then on, there have been crucial fields that signed the basis for neonatal care over the last century, such as thermoregulation, Apgar score, respiratory support, prenatal corticosteroids, metabolic screening and jaundice.

\section{Thermoregulation}

At the beginning of the 20th century, neonatal mortality was high and the introduction of temperature regulation considerably reduced it. The invention of the incubator is associated with Stephane Tarnier, a French obstetrician who first tried to introduce a mean to contrast hypothermia that occurred routinely in numerous

Correspondence to:

Giuseppe Buonocore

Department of Molecular and Developmental Medicine, University of Siena, Italy.

Tel.: +39.057.7586542 - Fax: +39.057.7586182.

E-mail: buonocore@unisi.it 
premature infants who succumbed on the wards of Paris' Maternitè Hospital in the 1870s. He statistically demonstrated the significance of this device comparing premature infant mortality before and after the use of it. Results were remarkable, in 500 infants in the 1200 to $2000 \mathrm{~g}$ range mortality dropped from $66 \%$ to $38 \%{ }^{8} \mathrm{~A}$ lot of types of incubators were conceived and used in Europe and the United States, until Silverman, Day et al. in the 1950s at Columbia, New York, revealed the benefits of controlling body temperature in term of reducing metabolic rate, oxygen consumption and hypoglycemia. Today's incubators also have the function to protect premature babies from infection, allergens, excessive noise and light levels that can be detrimental. In addition, incubators have now been developed as a multifunctioning system that comprises several tasks as the supplementation of oxygen, provision of nutrition, monitoring of vital parameters, administration of medications and maintaining fluid balance. ${ }^{9}$

\section{Apgar score}

In the early 1950s, an obstetric anesthesiologist, Virginia Apgar, developed a reliable assessment of the newborn at birth. Apgar's system of evaluation was then presented in 1952 at the 27th annual Congress of Anesthetists and International College of Anesthetists and published in Anesthesia and Analgesia in 1953. ${ }^{10}$ Her system implies to evaluate the newborn using five parameters - heart rate, reflex activity, respiration, tone and color - within the first minute and reevaluation at 5 or, if necessary, 10 minutes. ${ }^{11}$ This method remains still today a valid tool in neonatal assessment around the world. ${ }^{12}$

\section{Respiratory support}

From the late 19th century to 1950 's ventilation was delivered to the body of the patient using sub-atmospheric pressure with the aim to replace or enhance respiratory muscles work. ${ }^{13}$ In 1950 Allan P. Bloxsom introduced a new device, the positive oxygen air lock, to resuscitate and oxygenate the asphyxiated newborn in the delivery room. This unit provided warmed humidified $60 \%$ oxygen. The naked newborn entire body was placed in the pressure lock if "failure to breathe or breathe properly" occurred. The positive pressure was cycled between zero and five pounds per square inch to replicate the intrauterine pressures during the second stage of labor. The infant remained in the pressure lock until respirations became established and the infant well oxygenated. This treatment led him to the conclusions that the most important mechanism for the initial onset of respiration was absorption of oxygen through the skin and upper respiratory tract and that oxygen plays a fundamental role for the prompt stimulation of the respiratory center. The randomized, controlled clinical trial of the positive pressure air lock was finally published in $1956 .{ }^{14,15}$ First attempts to provide continuous respiratory support through mechanical ventilation to infants with severe respiratory disease were made in the mid-1960s. At that age, most preterm infants died for respiratory distress syndrome (RDS) or hyaline membrane disease (HMD), as it was originally called. Since that was the apparent cause of death of infant son of President John F. Kennedy, research had been implemented to investigate the cause and management of the disorder. In 1959, Mary Ellen Avery and Jere Mead linked the pathogenesis of this disorder with the deficiency of surfactant in the lung. ${ }^{16}$ Over the past 60 years, new modes of ventilation have been developed and many technical aspects improved such as flow delivery, use of microprocessors and exhalation valves. In the 1990's controlled ventilation moved towards partial ventilation support and finally pressure support ventilation. ${ }^{13}$

\section{Prenatal corticosteroids}

The discovery of therapy for fetal maturation happened upon incidentally, when the obstetrician Graham Liggins was studying in a sheep model factors involved in the initiation of labor, testing his hypothesis that steroid hormones might trigger labor. $\mathrm{He}$ found more mature lungs in preterm lambs exposed to corticosteroids in utero, that also were surviving at earlier gestational age and had milder respiratory distress. ${ }^{17}$ Then Liggins and Howie conducted a randomized controlled trial of maternal administration of betamethasone, showing a reduction of incidence of RDS in preterm infants and neonatal mortality. ${ }^{18}$ In 1990, a meta-analysis published by Crowley and colleagues showed that antenatal betamethasone has a protective effect against the development of also other neonatal morbidities such as intra- ventricular hemorrhage (IVH) and necrotizing enterocolitis (NEC). ${ }^{19}$

\section{Neonatal metabolic screening}

By the mid-20th century, recognition of the utility of systematic prevention, confirmed by polio vaccines and the identification and treatment of congenital syphilis, leaded to universal Neonatal Screening programs. ${ }^{20}$ The first test was called the Guthrie test and was introduced by Robert Guthrie, who developed a bacterial inhibition assay for phenylalanine together with the filter paper blood specimen system that made systematic screening possible. ${ }^{21}$ Over the next decade, several other inborn errors of metabolism were added to the screening, such as maple-syrup urine disease, homocystinuria, galactosemia and congenital hypothyroidism, introduced in the early 1970s. Recently, with the development of electrospray tandem mass spectrometry, a single test is used to screen for a wide range of very rare disorders that have not been screened for previously. ${ }^{22}$

Jaundice: Over the past $40 \mathrm{y}$, there has been an enormous change in the cause of severe hyperbilirubinemia. Rhesus incompatibility was a major problem 40 years ago, resulting in the need to perform large num- 
bers of exchange transfusions to prevent the development of kernicterus, whereas now has been virtually eradicated. ${ }^{23}$ The mainstay of treatment is phototherapy. Since early discharge of term infants has become the norm, detecting and preventing severe hyperbilirubinemia makes the task of more difficult. The use of transcutaneous bilirubinometry to facilitate detection and lessens the need for blood tests. Norms for agespecific levels of total serum bilirubin, hour by hour from 18 to $168 \mathrm{~h}$ after birth have been published. ${ }^{24}$

All these discoveries and advances in neonatology have resulted in the reduction of infant mortality worldwide. A major contributor to the decline in infant mortality is the decline in neonatal deaths associated with low birthweights; in fact, neonatal survival or mortality is strongly related to the number of premature or LBW infants born. In the United States, the incidence of prematurity has increased in recent years: this is largely due to an increase in the number of multiple births, often the result of advances in assisted reproduction techniques that now are commonplace. Prematurity is responsible for $75 \%$ of perinatal mortality and more than $50 \%$ of long-term morbidity. ${ }^{25}$ However, an infant born in 1950 with a birthweight $<1000$ grams had only a $10-15 \%$ chance of survival, while an infant born in 2008 with a birthweight $<1000$ grams had a $>60 \%$ chance of surviving the neonatal period. The gestational age at which $50 \%$ of neonates survived decreased from 29 weeks (wks) in 1960 to 24 wks by the early 1990s. Unfortunately, the number of infants with disabilities has stayed approximately stable over time because of increased survival at lower gestational ages. ${ }^{26}$ The majority of infants born very preterm now survive, but short-term consequences (RDS, IVH, periventricular leukomalacia-PVL, early- and late-onset sepsis, NEC) and long-term consequences (respiratory morbidity linked to bronchopulmonary dysplasia-BPD, visual impairments related to retinopathy of prematurity-ROP, neurodevelopmental and behavioral problems) remain a concern. With prematurity, the other leading causes of neonatal mortality and neurological disability are cerebral stroke and hypoxic-ischemic encephalopathy (HIE), more frequent in full-term babies. Therefore, today and in the future, further efforts must be made to improve neonatal and longterm outcomes. ${ }^{27}$

\section{WHAT ABOUT TODAY?}

To date, scientific evidences have shown that the environmental conditions experienced in early life can profoundly influence human biology and long-term health. This concept underlies the emerging interest toward the developmental care for preterm newborns, a range of strategies designed to reduce the stresses of the Neonatal Intensive Care Units (NICU). An unfavorable environment in the NICU can add to the prematu- rity-related problems and negatively affect the infant's growth, with the brain being particularly vulnerable; the preterm brain is in fact susceptible to a stressful environment and the detrimental effects of this stress could have short and long-term implications for compromised neurobehavioral development. Environmental modifications like reducing noise and light, minimal handling and giving longer rest periods, together with adequate pain control, could minimize the iatrogenic effects on the future health of the infant. ${ }^{28}$

However, another aspect of the early environmental impact on the future health of the newborn has been extensively studied in the last decades. More and more studies have confirmed the Barker's hypothesis or thrifty phenotype hypothesis, according to which environmental factors as poor nutrition in early life produces permanent changes in glucose-insulin metabolism and so predisposes to chronic adult diseases like metabolic syndrome and cardiovascular disease. ${ }^{29}$ The concept of fetal programming has long been known: fetal programming occurs when the normal fetal development is disrupted by an abnormal insult applied to a critical point in intrauterine life; placenta assumes a crucial role in programming the intrauterine experience due to the adaptive changes in structure and function and so a crucial role in developmental plasticity. ${ }^{30}$ Many complications of gestation such as preeclampsia, gestational diabetes and hypoxia can affect fetal programming, and recently an emerging area of research is the diet-induced inflammation in gestational tissues on fetal growth and development. Accumulating evidences now suggest that low-grade intrauterine inflammation might impair linear growth and adversely affect myogenesis and adipogenesis that might have lasting effects on offspring. ${ }^{31}$ Epigenetic modulation represents the molecular mechanism that underlies the development of adverse health conditions in adulthood. In the past, it was thought that at the base of the development of many pathological conditions there were predominantly non-modifiable genetic factors, now we are instead aware of the importance of environmental factors (diet, toxic, drugs, microbiome, social and financial status and so on) that act through epigenetic modifications. These changes, especially occurring in the early period of life that is the one of major plasticity, contribute to the individual susceptibility to disease and can lead to transgenerational detrimental effects. Maternal nutritional constraint during pregnancy can alter the metabolic phenotype of the offspring by means of epigenetic regulation of specific genes, and this can be passed to the next generations, ${ }^{32}$ but many other factors - maternal, fetal and placental - can influence the future vulnerability of the offspring. In a recent review, Feinberg argued that epigenetic changes are involved in normal development and human disease and proposed the term "epigenetic disease" to describe defects in the epigenome that are known to lead to disease. These de- 
fects include changes in the localized or global density of DNA methylation, incorrect histone modifications or altered distribution or function of chromatin-modifying proteins that, in turn, lead to aberrant gene expression. According to Feinberg, defects in phenotypic plasticity or the cell's ability to change its behavior in response to internal or external environmental cues are the underlying theme of epigenetic disease; this can also be applied to common diseases with late-onset phenotypes that involve interactions between the epigenome, the genome, and the environment. ${ }^{33}$

According to recent research, the lowest common denominator seems to be the oxidative stress (OS). Chemical contaminants in water and diet, tobacco smoke, air pollution, gestational diabetes, hypertension and preeclampsia are all conditions that can produce an imbalance in the pro-oxidant/anti-oxidant system, leading to the increased production of free radicals (FRs) during gestation and so to fetal oxidative stress. Fetuses and newborns - especially preterm - are particularly susceptible to such an imbalance because of their exposure to conditions that can lead to a burden of FRs (ischemia, hypoxia-reperfusion, infections, inflammation, but also transfusions, drugs, hyperoxia) in association with insufficient scavenger systems. ${ }^{32}$ FRs are highly reactive substances capable to start self-amplified chain reactions causing cellular dysfunction and damage to all components of the cell, including protein, lipids and DNA. ${ }^{34}$ Biomarkers in vivo of oxidative damage are non protein-bound iron (NPBI, marker of potential OS), Advance Oxidation Protein Product (AOPP, marker of protein oxidation), isoprostanes (IsoPS, markers of lipid peroxidation deriving from arachidonic acid) and Isofurans (IsoFs, metabolically stable compounds forming at higher oxygen tension), Neuroprostanes (NPs, markers of neuronal oxidative damage deriving from lipid oxidation of docosahexaenoic acid-DHA) and Neurofurans (NFs). ${ }^{34,35}$

Many known or suspected causes of or conditions associated with impaired fetal growth or preterm birth have been associated with OS, so it may be the common link underlying the associations between adverse fetal growth or preterm birth and elevated risks of certain chronic diseases. The mechanisms of "oxidative stress programming" may be direct, through modulating gene expression, or indirect, through the effects of the oxidized molecules. ${ }^{36}$ Some conditions of pregnancy are specific trigger for the overload of FRs: preeclampsia, intrauterine growth restriction, diabetes and maternal obesity. In fact F2-Isoprostanes (F2-IsoP), the main marker of arachidonic acid peroxidation, are higher in pregnancies with fetal growth restriction compared with pregnancies without, when dosed in amniotic fluid; they have a moderate power to distinguish fetal-growth-restricted pregnancies and so between adeguate- and small-for-gestational-age newborns. ${ }^{37}$ Furthermore, also preterm premature rupture of membranes (pPROM) has been associated with
OS, demonstrated by higher levels of F2-IsoP in amniotic fluid of mothers with pPROM compared with control pregnancies. ${ }^{38}$ In the preterm neonate, tissue and organ damage involving kidneys, retina, lung, brain, and bowel has been related with elevated level of OS biomarkers in cord blood. In a recent study, the development of pathologies of prematurity like ROP, BPD, NEC and IVH was significantly associated with high cord blood levels of Total Hydroperoxide- $\mathrm{TH}$, AOPP, and NBPI, thus leading to the hypothesis of "Free Radical-related Disease" (FRD) of prematurity. ${ }^{38}$ In particular, plasma NBPI has demonstrated to be the best early predictive marker of neonatal brain damage, with a $100 \%$ sensitivity and $100 \%$ specificity for good neurodevelopmental outcome at 0-1.16 micro mol/L, and for poor neurodevelopmental outcome at values $>15.2 \mathrm{micro} \mathrm{mol} / \mathrm{L}^{38}$

Not only preterm, but also full-term babies are particularly vulnerable to OS-related damage: OS seems to represent the underlying pathological mechanism even in neonatal asphyxia. In a recent study, newborns with severe asphyxia showed higher OS than those with mild asphyxia at birth, and AOPP was significantly associated with the severity of brain injury assessed by neuroimaging techniques (MRI), especially in males. The presence of an association between biomarkers of OS measured in the first hours of life and brain damage successfully evaluated through neuroimaging emphasizes the possibility of early identification of newborns at greater risk of brain damage. ${ }^{38}$

All these evidences open the door to the ever-increasing research on antioxidant strategies. There are many antioxidants studied, especially for neonatal brain injury: iron chelators, FRs scavengers, inhibitors of lipid peroxidation, FRs reducing agents and many others. Among these, melatonin and docosahexaenoic acid are particularly manageable and promising according to studies on animal models, although further confirmations on humans are necessary. ${ }^{39-42}$

\section{WHAT DOES THE FUTURE HOLD?}

Many initiatives are already part of the present, but represent the future since will allow the increasing improvement of the quality of life when they arrive at the patient's bedside.

Some example are gene therapy that will consent to replace a defective gene that underlies a specific condition, or biologic reporters, light-emitting enzymes (luciferases) that can label genes and cells and could be used to monitor gene expression and immune therapies. ${ }^{43}$

The "omics" sciences represent perhaps the major area of growing interest and research in neonatology and paediatrics. Genomics, transcriptomics, proteomics and metabolomics represent all the complexity of biological systems and are going to replace traditional laboratory 
methodologies thanks to their capacity to distinguish a single subject in normal conditions and in case of disease with a simultaneous non-invasive analysis of a large amount of data. ${ }^{39}$ Metabolomics summarizes the gene-environment interactions, and consists of the quantitative analysis of a large number of low molecular mass metabolites involving substrates or products in metabolic pathways existing in all living systems. The analysis of the metabolic profile detectable in a human biological fluid (urine, plasma, also cord blood plasma, milk, stool...) allows to instantly identifying changes in the composition of endogenous and exogenous metabolites caused by the interaction between specific physiopathological states, gene expression, and environment. ${ }^{44,45}$ From this point of view, metabolomics represent a sort of "identity card" of the individual in normal and pathological conditions, with each condition or disease presenting a specific discriminating set of metabolites.

In a recent study, distinct metabolic patterns were found between term infants and preterm infants, as well as between preterm infants of 23-32 week' gestation and those of 33-36 weeks' gestation. Individual metabolites discriminating between these groups were Hippurate, tryptophan, phenylalanine, malate, tyrosine, hydroxybutyrate, $\mathrm{N}$-acetyl-glutamate and proline. Metabolomic analysis revealed distinct urinary profiles in newborns of different gestational ages and identified the discriminating metabolites. ${ }^{46}$ In all sectors of the neonatology field there are ongoing studies aimed at validating the metabolic profiles correlated to specific neonatal pathological conditions, like neonatal asphyxia and hypoxic-ischemic encephalopathy, sepsis, necrotizing enterocolitis, acute kidney injury, bronchopulmonary dysplasia. For example, the urinary metabolic profile of newborns with HIE results significantly different from the one of healthy newborns. ${ }^{46}$

These recent advances lead to the concept of an individualized and tailored medicine. The application of omics methodologies in paediatrics and neonatology is crucial due to its unique ability to generate functional readouts of biological systems, but the clinical translation of this source of knowledge into clinical practices for neonatal health care requires proper addressing of the inherent inter-individual variability. ${ }^{46}$ Therefore, further studies on this subject are certainly necessary but we can affirm with certainty that metabolomics is really the future, above all for our infants.

Remember: "As neonatologists, we owe an enormous debt to our predecessors, but we are still faced with many challenges for the future"[A.G.S. Philip].

\section{ACKNOWLEDGMENTS}

The authors thank EURAIBI (EURope Against Infant Brain Injury) Foundation for its support.

\section{REFERENCES}

1. BBC Four. Medieval Lives: Birth, Marriage and Death. 2013. Available from: https://www.bbc.co.uk/programmes/b03czjbq

2. Green MH. The sources of Eucharius Rosslin's 'Rosegarden for pregnant women and midwives' (1513). Med Hist 2009;53:167-92.

3. Epstein RH. Get me out. A history of childbirth form the garden of Eden to the sperm bank. New York: W.W. Norton $\theta$ Company; 2010 .

4. Philip AG. The evolution of neonatology. Pediatr Res 2005:58.

5. Baker JP. The incubator and the medical discovery of the premature infant. J Perinatol 2000;20:321-8.

6. Parmelee AH. Management of the Newborn. Chicago: Year Book Publishers; 1952.

7. Schaffer AJ. Diseases of the Newborn. Philadelphia: W.B. Saunders; 1960

8. Auvard A. De la couveuse pour enfants. Paris: Delahaye; 1883.

9. Whitfield JM, Peters BA, Shoemaker C. Conference summary: a celebration of a century of neonatal care. Proc Bayl Univ Med Cent 2004; 17:255-8.

10. Apgar V. A proposal for a new method of evaluation of the newborn infant. Curr Res Anesth Analg 1953;32:260-7.

11. Apgar V, Holaday DA, James LS, et al. Evaluation of the newborn infant; second report. JAMA 1958;168:1985-8.

12. Casey BM, McIntire DD, Leveno KJ. The continuing value of the Apgar score for the assessment of newborn infants. N Engl J Med 2001;344:467-71.

13. Slutsky AS. History of Mechanical Ventilation. From Vesalius to Ventilator-induced Lung Injury. Am J Respir Crit Care Med 2015;191:1106-15.

14. Kendig JW, Maples PG, Maisels MJ. The Bloxsom Air Lock: A Historical Perspective. Pediatrics 2001;108:E116.

15. Zaichkin J, Wiswell TE. The History of Neonatal Resuscitation. Neonatal Netw 2002;21:21-8.

16. Avery ME, Mead J. Surface properties in relation to atelectasis and hyaline membrane disease. Am J Dis Child 1959;97:517-23.

17. Bonanno C, Wapner RJ. Antenatal Corticosteroids in the Management of Preterm Birth: Are we back where we started? Obstet Gynecol Clin North Am 2012;39:47-63.

18. Liggins GC, Howie RN. A controlled trial of antepartum glucocorticoid treatment for prevention of the respiratory distress syndrome in premature infants. Pediatrics 1972;50:515-25.

19. Crowley P, Chalmers I, Keirse MJ. The effects of corticosteroid administration before preterm delivery: an overview of the evidence from controlled trials. Br J Obstet Gynaecol 1990;97:11-25.

20. Brosco JP, Seider MI, Dunn AC. Adverse Medical Outcomes of Early Newborn Screening Programs for Phenylketonuria. Pediatrics 2006;269:262-9.

21. Dhondt J. Neonatal screening: from the F Guthrie age to the F genetic age. $J$ Inherit Metab Dis 2007;30:418-22.

22. Wilcken B, Wiley V, Carpenter K. Screening newborns for inborn errors of metabolism by tandem mass spectrometry. N Engl J Med 2003;348:2304-12.

23. Maisels MJ, Kring E. Length of stay, jaundice, and hospital readmission. Pediatrics 1998;101:995-8.

24. No authors listed. Rh-disease a perinatal success story. Obstet Gynecol 2002;100:405-6.

25. Blencowe H, Cousens S, Oestergaard MZ, et al. National, regional, and worldwide estimates of preterm birth rates in the year 2010 with time trends since 1990 for selected countries: a systematic analysis and implications. Lancet 2016;379:2162-72.

26. Glass HC, Costarino AT, Stayer SA, et al. Outcomes for extremely premature infants. Anesth Analg 2015;120:1337-51.

27. Institute of Medicine (US) Committee on Understanding Prema- 
ture Birth and Assuring Healthy Outcomes. Preterm Birth: Causes, Consequences, and Prevention. Washington (DC): National Academies Press (US); 2007.

28. Symington A, Pinelli J. Developmental care for promoting development and preventing morbidity in preterm infants. Cochrane Database Syst Rev 2001:CD001814.

29. Hales CN, Barker DJ. The thrifty phenotype hypothesis. Br Med Bull 2001;60:5-20.

30. Perrone S, Santacroce A, Picardi A, Buonocore G. Fetal programming and early identification of newborns at high risk of free radical-mediated diseases. World J Clin Pediatr 2016;5:172-81.

31. Hemalatha R. Diet Induced Inflammation and Potential Consequences on Fetal Development. J Nutr Disord Ther 2013;3:125.

32. Burgio E, Lopomo A, Migliore L. Obesity and diabetes: from genetics to epigenetics. Mol Biol Rep 2015;42:799-818.

33. Feinberg AP. Phenotypic plasticity and the epigenetics of human disease. Nature 2007;447:433-40.

34. Perrone S, Santacroce A, Longini M, et al. The Free Radical Diseases of Prematurity: from cellular mechanisms to bedside. Oxid Med Cell Longev 2018;2018:7483062.

35. Buonocore G, Perrone S, Tataranno ML. Oxygen toxicity: chemistry and biology of reactive oxygen species. Semin Fetal Neonatal Med 2010;15:186-90.

36. Luo ZC, Fraser WD, Julien P, et al. Tracing the origins of "fetal origins" of adult disease: programming by oxidative stress? Med Hypotheses 2006;66:38-44.

37. Longini $M$, Perrone S, Kenanidis A, et al. Isoprostanes in amniotic fluid: a predictive marker for fetal growth restriction in pregnancy. Free Radic Biol Med 2005;38:1537-41.
38. Longini M, Perrone S, Vezzosi P, et al. Association between oxidative stress in pregnancy and preterm premature rupture of membranes. Clin Biochem 2007;40:793-7.

39. Solberg $R$, Longini $M$, Proietti $F$, et al. DHA reduces oxidative stress after perinatal asphyxia: a study in newborn piglets. Neonatology 2017;112:1-8.

40. Signorini C, Ciccoli L, Leoncini S, et al. Free iron, total F-isoprostanes and total F-neuroprostanes in a model of neonatal hy poxic-ischemic encephalopaty: neuroprotective effect of melatonin. J Pineal Res 2009;46:148-54.

41. Tataranno ML, Perrone S, Longini M, Buonocore G. New antioxidant drugs for neonatal brain injury. Oxid Med Cel Longev 2015;108251.

42. Perrone S, Stazzoni G, Tataranno ML, Buonocore G. New pharmacologic and therapeutic approaches for hypoxi-ischemic encephalopaty in the newborn. J Matern Fetal Neonatal Med 2012;25:83-8.

43. McCaffrey A, Kay MA, Contag CH. Advancing molecular therapies through in vivo bioluminescent imaging. Mol Imaging 2003;2:75-86.

44. Mussap M, Antonucci $R$, Noto A, Fanos $V$. The role of metabolomics in neonatal and pediatric laboratory medicine. Clin Chim Acta 2013;426:127-38.

45. Buonocore G, Mussap M, Fanos V. Proteomics and metabolomics: can they solve some misteries of the newborn? J Matern Fetal Neonatal Med 2013;26:7-8.

46. Atzori L, Antonucci $R$, Barberini L, et al. $1 H$ NMR-based metabolomic analysis of urine from preterm and term neonates. Front Biosci (Elite Ed) 2011;3:1005-12. 